\title{
Hubble Space Telescope ultraviolet spectroscopy of the hottest known helium-rich pre-white dwarf KPD 0005+5106
}

\author{
K. Werner and T. Rauch \\ Institute for Astronomy and Astrophysics, Kepler Center for Astro and Particle Physics, Eberhard Karls University, Sand 1, \\ 72076 Tübingen, Germany \\ e-mail: werner@astro.uni-tuebingen.de
}

Received 19 August 2015 / Accepted 21 September 2015

\begin{abstract}
We present a model-atmosphere analysis of the ultraviolet echelle spectra of KPD 0005+5106 taken with the Space Telescope Imaging Spectrograph aboard the Hubble Space Telescope. The star is the hottest known pre-white dwarf $\left(T_{\text {eff }}=200000 \pm 20000 \mathrm{~K}, \log g=\right.$ $\left.6.7 \pm 0.3\left(\mathrm{~cm} / \mathrm{s}^{2}\right)\right)$. Its atmosphere is composed of helium with trace amounts of metals. It is of the so-called $\mathrm{O}(\mathrm{He})$ spectral type that comprises very hot helium-rich pre-white dwarfs whose origin is debated. From neon and silicon ionisation balances, we derive tighter constraints on the effective temperature $(195000 \pm 15000 \mathrm{~K})$ and improve previous abundance determinations of these elements. We confirm the idea that KPD 0005+5106 is the descendant of an R Coronae Borealis (RCB) star, so is the outcome of a binary-whitedwarf merger. We discuss the relation of KPD $0005+5106$ to other $\mathrm{O}(\mathrm{He})$ and $\mathrm{RCB}$ stars.
\end{abstract}

Key words. stars: abundances - stars: atmospheres - stars: evolution - stars: AGB and post-AGB - white dwarfs

\section{Introduction}

KPD $0005+5106$ is the hottest known pre-white dwarf star. It has a helium-dominated atmosphere with trace metals and no detectable hydrogen $(\mathrm{H}<2.5 \%$ mass fraction; Wassermann et al. 2010). It was originally classified as a hot DO white dwarf (WD) with an effective temperature of $T_{\text {eff }}=120000 \mathrm{~K}$ and a surface gravity of $\log g=7\left[\mathrm{~cm} / \mathrm{s}^{2}\right]$ (Werner et al. 1994), however, it was realized later that the star is much hotter and has a lower surface gravity $\left(T_{\text {eff }}=200000 \pm 20000 \mathrm{~K}, \log g=6.7 \pm 0.3\right)$, i.e., higher luminosity, meaning that it actually is still helium-shell burning (Werner et al. 2007). Consequently, it must be classified as an $\mathrm{O}(\mathrm{He})$ star, a designation introduced by Mendez (1991) for helium-dominated pre-white dwarfs with spectra characterized by absorption lines from ionized helium.

The origin of the $\mathrm{O}(\mathrm{He})$ stars is unclear and currently debated (e.g., Reindl et al. 2014). The majority of the pre-white dwarfs are rich in hydrogen and compatible with canonical stellar evolution theory (e.g., Iben 1995). A large number are, however, hydrogen-deficient, and most of these objects are PG 1159 stars that are also helium-rich but, in contrast to the $\mathrm{O}(\mathrm{He})$ stars, also strongly enriched in carbon and oxygen. The PG 1159 surface chemistry is explained by a late helium-shell flash that consumes the hydrogen envelope and dredges up helium, enriched by Heburning ashes from the stellar core (Iben et al. 1983; Herwig et al. 1999; Werner \& Herwig 2006). It has been argued that the $\mathrm{O}(\mathrm{He})$ stars represent a distinct post-AGB sequence, possibly initiated by binary WD mergers, and it was speculated that they are descendants of the R Coronae Borealis (RCB) stars. This evolutionary link was invoked particularly for KPD 0005+5106 because its trace element abundances are rather similar (Rauch et al. 2008; Wassermann et al. 2010).

RCB stars are hydrogen-deficient supergiants (Clayton 1996 ) with $T_{\text {eff }}=4000-8000 \mathrm{~K}$ and $\log g=0.5-1.5$. There is now general consensus that RCB stars have a binary WD merger origin (Webbink 1984), based on evidence from evolution timescales, pulsation masses, and surface element abundances (Saio \& Jeffery 2002; Pandey et al. 2006; Clayton et al. 2007). Closely related are the Extreme Helium stars (EHe), which are hotter, early-type (A and B) supergiants with similar photospheric composition, and they are assumed descendants of the RCB stars (e.g., Jeffery et al. 2011).

The abundance pattern of KPD $0005+5106$ was derived by the analysis of optical but mainly far-ultraviolet spectra taken with the Far-Ultraviolet Spectroscopic Explorer (FUSE), covering the wavelength range 912-1180 (Rauch et al. 2008; Wassermann et al. 2010). In this paper, we present new ultraviolet (UV) spectra taken with the Hubble Space Telescope (HST), stretching the observed spectral range up to the optical. Our observations were intended to independently check for the effective temperature determination and to improve the determination of trace element abundances to better constrain the relation of KPD $0005+5106$ to the RCB stars and to shed more light on the evolutionary status of the $\mathrm{O}(\mathrm{He})$ stars.

We begin with a description of the observations (Sect. 2) and continue with a delineation of our model atmospheres and model atoms (Sect. 3) utilized for the spectral analysis. In Sect. 4 we present in detail the line identifications and line fitting procedure. Finally, the results are summarized and discussed in the context of $\mathrm{RCB}$ and $\mathrm{O}(\mathrm{He})$ stars in Sect. 5.

\section{Observations}

Short-slit $(0.2 \times 0.2)$, medium-dispersion UV spectroscopy of KPD $0005+5106$ in the STIS/FUV-MAMA and NUVMAMA configurations was performed with HST during Cycle 19, covering the wavelength range $\sim 1150-3100 \AA$ (Table 1). The spectral resolving power as listed in Table 1 corresponds to $\Delta \lambda=0.025-0.037 \AA$ in the $1144-1710 \AA$ range 
Table 1. Observation log of HST/STIS echelle spectroscopy.

\begin{tabular}{ccccc}
\hline \hline Dataset & Grating & $R$ & $\lambda / \AA$ & $t_{\exp } / \mathrm{s}$ \\
\hline OBON1010 & E230M & 30000 & $1616-2366$ & 300 \\
OBON1020 & E230M & 30000 & $2277-3072$ & 450 \\
OBON1030 & E140M & 45800 & $1144-1710$ & 1215 \\
OBON1040 & E140M & 45800 & $1144-1710$ & 3193 \\
\hline
\end{tabular}

Notes. Observations performed on March 16, 2012. Spectral resolving power is $R=\lambda / \Delta \lambda$. The last two columns give the wavelength range covered and the exposure times, respectively.

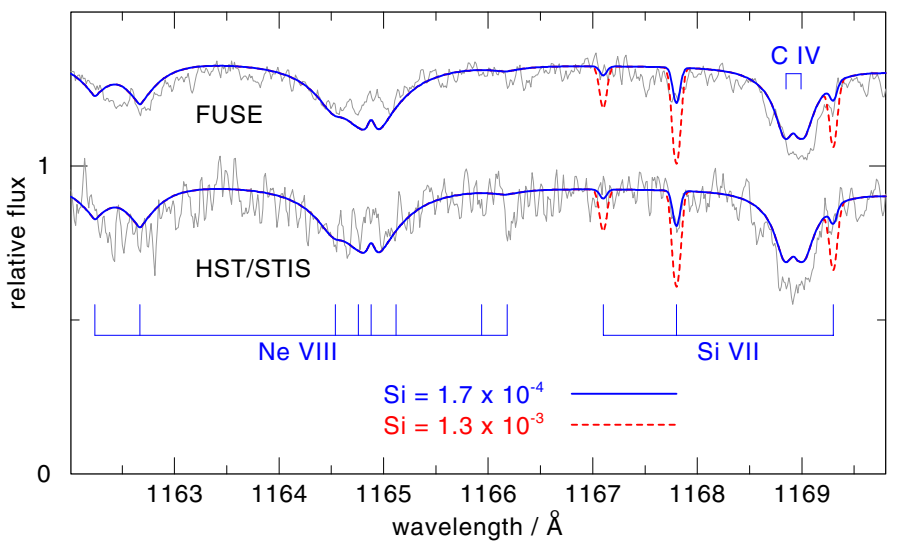

Fig. 1. Detail of the HST/STIS spectrum of KPD 0005+5106 (bottom, thin gray line) and the FUSE spectrum (top, thin gray line) comprising a Si VII triplet. Overplotted on each are two models (thick solid and dashed lines) with different $\mathrm{Si}$ abundances as indicated in the legend. Other model parameters: $T_{\text {eff }}=200000 \mathrm{~K}, \log g=6.7, \mathrm{C}=9.3 \times 10^{-3}$, $\mathrm{Ne}=3.9 \times 10^{-3}$ (mass fractions). Observations and models were convolved with $0.02 \AA$ boxcars and $0.036 \AA$ Gaussians, respectively.

and $\Delta \lambda=0.054-0.102 \AA$ in the $1616-3072 \AA$ range. The signalto-noise ratio $(\mathrm{S} / \mathrm{N})$ of the far-UV spectrum below $\sim 1190 \AA$ is relatively poor such that the quality of the co-added archival FUSE spectra in that wavelength range is better (see Fig. 1 for a comparison). Identified and unidentified photospheric lines are listed in Table 2.

Previous UV observations in this spectral range were performed with the International Ultraviolet Explorer (IUE, Downes et al. 1987) but the high-resolution spectra have S/N that is too low to detect weak metal lines. HST data were recorded with the Faint Object Spectrograph (FOS) with a low resolution of 2-4 $\mathrm{A}$ (Sion \& Downes 1992; Werner et al. 1994), also preventing the detection of weak lines. The same holds for spectra of similar resolution taken with the Hopkins Ultraviolet Telescope (HUT, Kruk \& Werner 1998). HST spectra with higher resolution were obtained with the Goddard High Resolution Spectrograph (GHRS); however, only a few selected, about $35 \AA$ wide wavelength intervals were recorded (Werner et al. 1996).

\section{Model atoms and model atmospheres}

For the spectral analysis we used our non-LTE code $^{1}$ (Werner et al. 2012) to compute plane-parallel line-blanketed atmosphere models in radiative and hydrostatic equilibrium (Werner \& Dreizler 1999; Werner et al. 2003). They include helium and the four most abundant trace elements (according to the results

\footnotetext{
1 http://astro.uni-tuebingen.de/ TMAP
}

Table 2. Photospheric lines detected in the HST/STIS spectra.

\begin{tabular}{|c|c|c|c|}
\hline Wavelength/Å & Ion & Transition & \\
\hline $1162.24,1162.67$ & Ne VIII & $5 d-6 f$ & \\
\hline $1164.54,1164.76$ & Ne VIII & $5 f-6 g$ & \\
\hline 1164.88 & Ne VIII & $5 g-6 h$ & \\
\hline 1165.94:, 1166.15:, 1166.18: & Ne VIII & $5 f-6 d$ & \\
\hline $1167.8,1169.3$ & Si VII & $3 s^{3} S_{*}^{o}-3 p{ }^{3} P_{*}$ & \\
\hline $1168.85,1168.99$ & C IV & $3 d-4 f$ & \\
\hline 1171.12:, 1172.00: & O VI & $4 p-5 s$ & \\
\hline $1198.55,1198.59$ & C IV & $3 d-4 p$ & \\
\hline 1207.68: & Si VI & $3 \mathrm{~s}^{2} \mathrm{D}_{5 / 2}-3 \mathrm{p}^{2} \mathrm{~F}_{7 / 2}^{\mathrm{o}}$ & \\
\hline $1230.04,1230.52$ & C IV & $3 p-4 s$ & \\
\hline 1236.0 & Si VII & $3 \mathrm{~s}^{\prime}{ }^{1} \mathrm{D}^{\mathrm{o}}-3 \mathrm{p}^{\prime}{ }^{1} \mathrm{~F}$ & \\
\hline $1238.82,1242.80$ & N V & $2 s-2 p$ & \\
\hline 1243.1 & $?^{b}$ & & \\
\hline 1287.80 & $?$ & & \\
\hline 1293.9: & Si VII & $3 \mathrm{~s}{ }^{1} \mathrm{P}^{\mathrm{o}}-3 \mathrm{p}{ }^{1} \mathrm{D}$ & \\
\hline 1315.62:, 1315.85: & CIV & $4 p-7 d$ & \\
\hline 1316.3 & $?$ & & \\
\hline 1317.7 & $?^{b}$ & & \\
\hline 1319.78 & Ne VII & $2 \mathrm{p}^{1} \mathrm{P}_{1}^{\mathrm{o}}-2 \mathrm{p}^{2}{ }^{3} \mathrm{P}_{2}$ & \\
\hline 1323.3 & $?$ & & \\
\hline $1351.21,1351.29$ & CIV & $4 d-7 f$ & \\
\hline 1352.97 & CIV & $4 f-7 g$ & \\
\hline 1382.1 & $?$ & & \\
\hline 1401.6 & $?^{b}$ & & \\
\hline 1429.1 & $?^{a}$ & & \\
\hline 1437.65 & $?^{b}$ & & \\
\hline $1440.30,1440.38$ & CIV & $4 s-6 p$ & \\
\hline $1441.7-1442.7$ & $?$ & & \\
\hline $1450.0,1450.55,1451.7$ & $?^{b}$ & & \\
\hline 1456.8 & $?^{a}$ & & \\
\hline $1457.85,1458.25$ & $?^{a}$ & & \\
\hline 1461.5 & $?$ & & \\
\hline 1480.2 & $?^{a}$ & & \\
\hline 1484.9 & $?^{b}$ & & \\
\hline $1548.20,1550.77$ & CIV & $2 s-2 p$ & \\
\hline $1548.67,1549.34,1549.45$ & $\mathrm{NV}$ & $4 p-5 d$ & \\
\hline $1585.81,1586.11,1586.14$ & CIV & $4 p-6 d$ & \\
\hline $1619.62,1619.74$ & $\mathrm{NV}$ & $4 f-5 g$ & em \\
\hline 1640.42 & He II & $2-3$ & \\
\hline $1931.96,1932.01,1932.04$ & Ne VIII & $6 \mathrm{~h}-7 \mathrm{i}$ etc. & em \\
\hline 1981.97, 1992.06, 1997.35 & $\mathrm{Ne}$ VII & $3 s^{3} S-3 p{ }^{3} P^{o}$ & \\
\hline 2070.92:, 2071.02:, 2071.06: & O VI & $5 g-6 h$ etc. & em \\
\hline 2161.2: & Ne VII & $3 \mathrm{~s}{ }^{1} \mathrm{P}^{\mathrm{o}}-3 \mathrm{p}{ }^{1} \mathrm{D}$ & em \\
\hline 2253.39 & He II & $3-10$ & \\
\hline 2306.90 & He II & $3-9$ & \\
\hline 2386.13 & He II & $3-8$ & \\
\hline $2405.17,2405.83,2405.93$ & CIV & $4 p-5 d$ & \\
\hline 2511.96 & He II & $3-7$ & \\
\hline $2525.02,2525.27$ & CIV & $4 d-5 f$ & \\
\hline 2530.74: & CIV & $4 f-5 g$ & \\
\hline 2698.52:, 2699.47: & CIV & $4 p-5 s$ & \\
\hline 2734.11 & He II & $3-6$ & \\
\hline $2820.7,2860.1$ & $\mathrm{Ne}$ VIII & $3 s-3 p$ & \\
\hline 2907.19 & C IV & $5 g-7 h$ & \\
\hline 2976.75 & Ne VIII & $7 \mathrm{i}-8 \mathrm{k}$ etc. & em \\
\hline 2982.19 & N V & $5 g-6 h$ etc. & em \\
\hline
\end{tabular}

Notes. “:” denotes uncertain detection, "?" unidentified line, "em” emission line. ${ }^{(a)}$ Also visible in archival HST spectra of the PG 1159 stars NGC 246 and H1504+65. ${ }^{(b)}$ Also visible in H1504+65.

of Wassermann et al. 2010): C, N, O, and Ne. Four more species ( $\mathrm{Mg}, \mathrm{Si}, \mathrm{S}, \mathrm{Ca}$ ) were investigated and treated one by one as trace elements, i.e., keeping the atmospheric structure fixed. In the same manner, an extended model atom for Ne was introduced, meaning that non-LTE population numbers were also computed for highly excited levels that were treated in LTE during the preceding model-atmosphere computations. Table 3 summarizes the number of considered non-LTE levels and radiative transitions 
Table 3. Number of levels and lines of model ions used for lineformation calculations of metals.

\begin{tabular}{cccccccc}
\hline \hline & IV & V & VI & VII & VIII & IX & X \\
\hline $\mathrm{C}$ & 54,295 & & & & & & \\
$\mathrm{~N}$ & & 27,99 & & & & & \\
$\mathrm{O}$ & & 12,16 & 54,291 & & & & \\
$\mathrm{Ne}$ & & & 8,9 & 103,761 & 77,506 & & \\
$\mathrm{Mg}$ & & 15,18 & 27,60 & 46,147 & 50,269 & & \\
$\mathrm{Si}$ & 25,59 & 45,193 & 61,138 & 55,239 & & \\
$\mathrm{~S}$ & & 39,107 & 25,48 & 38,120 & 38,117 & & \\
$\mathrm{Ca}$ & & & & & 1,0 & 15,23 & 25,126 \\
\hline
\end{tabular}

Notes. First and second numbers of each table entry denote the number of levels and lines, respectively. Not listed for each element is the highest ionization stage considered in the model atom that only comprises its ground state.

between them. All model atoms were built from the publicly available Tübingen Model Atom Database $\left(\mathrm{TMAD}^{2}\right.$ ), comprising data from different sources, namely Bashkin \& Stoner (1975), the databases of the National Institute of Standards and Technology (NIST $\left.{ }^{3}\right)$, the Opacity Project $\left(\mathrm{OP}^{4}\right.$, Seaton et al. 1994), CHIANTI $^{5}$ (Dere et al. 1997; Landi et al. 2013), as well as the Kentucky Atomic Line List ${ }^{6}$.

Interstellar lines were modeled with the program Owens (Hébrard et al. 2002; Hébrard \& Moos 2003).

\section{Line identifications and spectral fitting}

Our analysis builds on the results of Wassermann et al. (2010). We computed a basic model with their derived values for effective temperature $\left(T_{\mathrm{eff}}=200000 \mathrm{~K}\right)$, gravity $(\log g=6.7)$, and element abundances. The model spectrum was compared to the new HST data, and adjustments of the model parameters were made to improve the spectral line fits. In particular, we checked whether we can constrain $T_{\text {eff }}$ better than in the previous work $( \pm 20000 \mathrm{~K})$. We do not improve the gravity determination of Wassermann et al. (2010). It was based on a careful analysis of all He II lines in the UV and optical bands, including $\lambda 1640 \AA$. This line is also covered by the STIS spectra (Fig. 2); however, the line wings are distorted because of obvious problems with the pipeline data reduction that reveals artificial residuals near the limits of the echelle orders. Interestingly, however, the spectrum exhibits a weak central emission core in the line. Close inspection of the line depth on either side of that emission core reveals that the $200000 \mathrm{~K}$ model fits well and the $180000 \mathrm{~K}$ model fits marginally better. The $220000 \mathrm{~K}$ model can be excluded because the line profile becomes too deep.

Generally, most spectral lines in our HST spectra are fit well by the basic model but, as in Wassermann et al. (2010), we encountered problems with particular lines that are better fit with models with slightly different parameters ( $T_{\text {eff }}$, abundances). In the following we discuss line identifications and model fits as far as they revealed new information compared to previous work.

\footnotetext{
2 http://astro.uni-tuebingen.de/ TMAD

3 http://www.nist.gov/pml/data/asd.cfm

4 http://cdsweb.u-strasbg.fr/topbase/topbase.html

5 http://www. chiantidatabase.org

6 http://www.pa.uky.edu/ peter/atomic
}

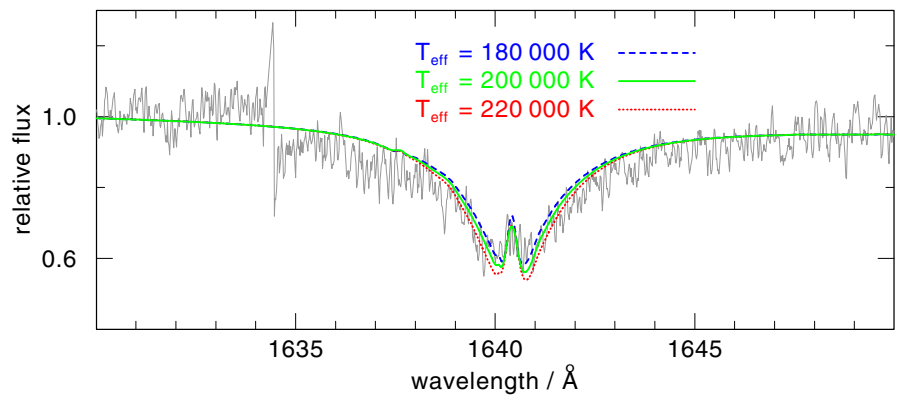

Fig. 2. He II $1640 \AA$ line in the HST/STIS spectrum (thin gray line). Overplotted are three models (thick lines) with different $T_{\text {eff }}$ (dashed, solid, and dotted, as indicated in the legend) and $\log g=6.7$. Observation and model were convolved with a $0.02 \AA$ boxcar and a $0.036 \AA$ Å Gaussian, respectively.

\subsection{Carbon, nitrogen, and oxygen}

We see lines from CIV that are well known from PG 1159 stars, but they are significantly weaker in KPD $0005+5106$ because of the lower carbon abundance. The abundance derived by Wassermann et al. $(2010, C=0.01)^{7}$ was a compromise because not all CIV lines in the optical and UV spectra could be fitted with the same value. We adopted that value for our models and kept it fixed. Two examples for lines that are too weak in the model are those at 1169 and $1230 \AA$ presented in Figs. 1 and 3.

From nitrogen we see, as previously detected in HST spectra, the N V resonance doublet at $1239 / 1243 \AA$, blended by stronger blueshifted interstellar components, as well as the N v $5 \mathrm{~g}-6 \mathrm{~h}$ emission line at $2982.18 \AA$. We confirm the formerly determined abundance of $\mathrm{N}=2.5 \times 10^{-3}$.

Oxygen lines are not present. It is remarkable that the O V $1371 \AA$ line is not detected because it confirms that $T_{\text {eff }}$ is at least $180000 \mathrm{~K}$. At this temperature, the $\mathrm{O}$ VI $5 \mathrm{~g}-7 \mathrm{~h}$ line at $1291 \AA$ would still be detectable, and only a model with $200000 \mathrm{~K}$ or higher is compatible with the absence of this line in the observation. For our modeling, we chose the abundance derived by Wassermann et al. (2010): $\mathrm{O}=4 \times 10^{-3}$.

\subsection{Neon}

The presence of Ne VIII lines in KPD $0005+5106$ was noted by Werner et al. (2007). Assuming $T_{\text {eff }}=200000 \mathrm{~K}$ and $\log g=7$, an abundance of $\mathrm{Ne}=1 \%$ was derived from a fit to the lines at $1162-1166 \AA$. Wassermann et al. (2010) arrived at a slightly lower abundance of $\mathrm{Ne}=0.4 \%$ after a detailed re-analysis that also gave a lower gravity, $\log g=6.7$. Figure 1 shows a fit to these lines with $\mathrm{Ne}=0.39 \%$ in the model.

In the new HST spectra, additional neon lines can be assessed. From Ne VII, we identify the strongest component of an intercombination triplet at $1320 \AA$ in absorption. Also, we see the triplet at 1982/1992/1997 $\AA$ in absorption. A weak emission feature near $2161 \AA$ can be attributed to a Ne VII singlet. Some more Ne VII lines are covered by the STIS spectra, but their wavelength positions are not well known (see line list in Reader et al. 2015, and uncertainty estimates in the Kentucky database). Our synthetic spectra predict the presence of such lines at positions computed from energy levels. Because of uncertainties in the energies, the line positions are uncertain by several $\AA$. Some of the unidentified lines, therefore, could well stem from Ne VII.

7 All abundances given in mass fractions unless otherwise noted. 


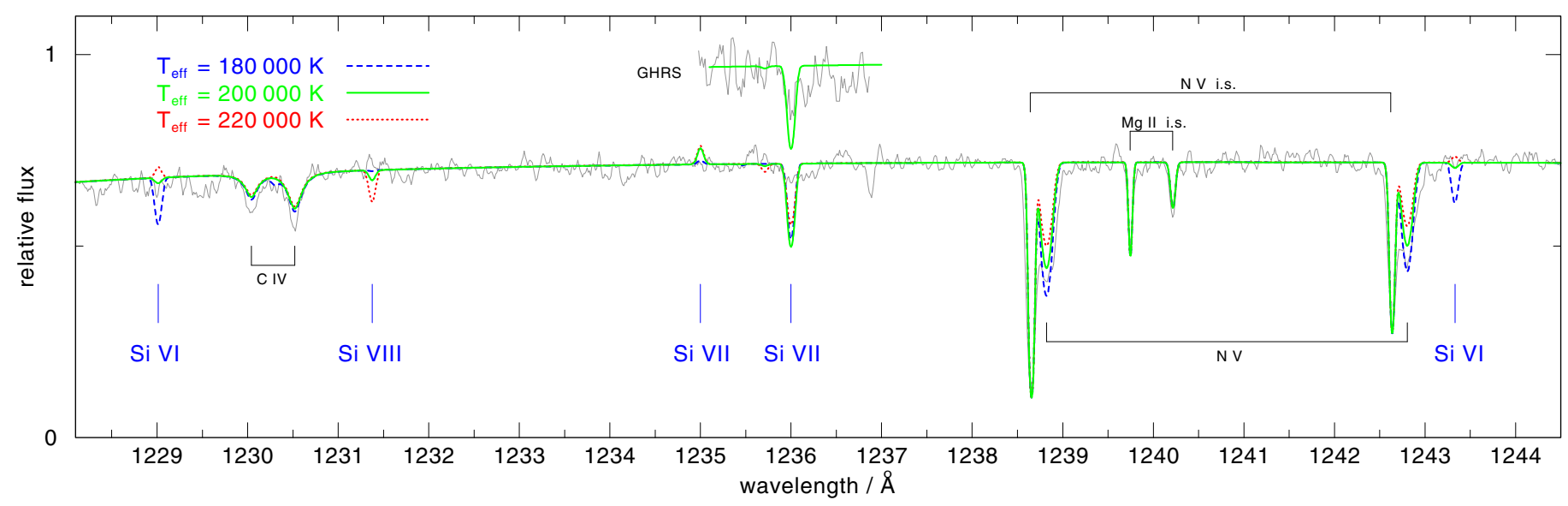

Fig. 3. Section of the HST/STIS spectrum (thin gray line) comprising silicon lines from three ionization stages. Overplotted are three models (thick lines) with different $T_{\text {eff }}$ (dashed, solid, and dotted, as indicated in the legend). At $180000 \mathrm{~K}$, the Si VI lines are too strong; the Si VIII line is too strong at $220000 \mathrm{~K}$. Other model parameters: $\log g=6.7, \mathrm{C}=9.3 \times 10^{-3}, \mathrm{~N}=2.2 \times 10^{-3}, \mathrm{Si}=1.3 \times 10^{-3}$. Also shown is the archival GHRS spectrum near the Si VII $1236 \AA$ line, shifted upward. Observations and models were convolved with $0.02 \AA$ boxcars and $0.036 \AA$ Gaussians, respectively.

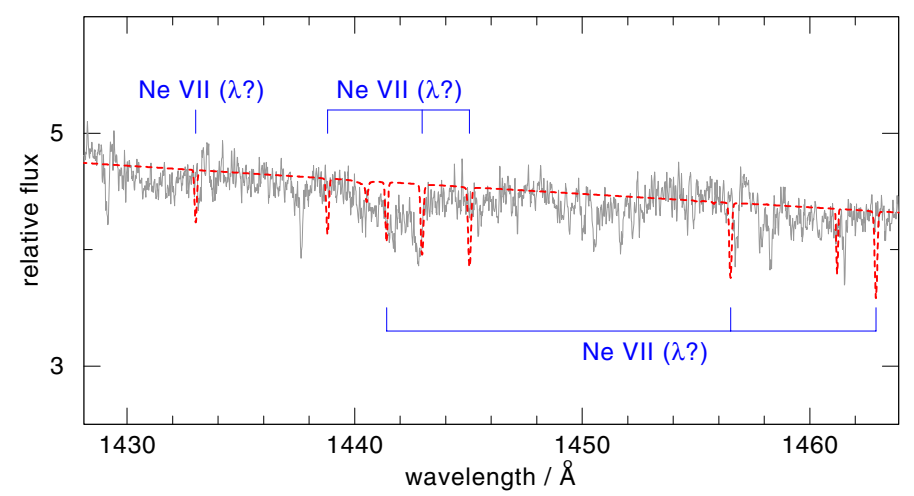

Fig. 4. Section of the HST/STIS spectrum (thin gray line) and a model (thick dashed line) comprising two Ne VII triplets and a singlet with uncertain wavelength positions. Some of the unidentified lines might correspond to the computed ones. Model parameters: $T_{\text {eff }}=200000 \mathrm{~K}$, $\log g=6.7, \mathrm{Ne}=1.2 \times 10^{-2}$. Observation and model were convolved with a $0.02 \AA$ boxcar and a $0.036 \AA$ Gaussian, respectively.

In Fig. 4 we show a region where two Ne VII triplets and a singlet are located in the model and where several unidentified lines in the observation are seen. As to Ne VIII, an emission feature near $1932 \AA$ is detected, and it was previously identified in the HST/FOS spectrum as an unresolved triplet (Werner et al. 2007). A rather strong Ne VIII doublet is present at 2821/2861 $\AA$. The following conclusions can be drawn on $T_{\text {eff }}$ and $\mathrm{Ne}$ abundance.

With models containing $\mathrm{Ne}=1 \%$, the Ne VII lines fit well at $180000 \mathrm{~K}$, while at $200000 \mathrm{~K}$, the $1320 \AA$ line is too weak, and the $1982 / 1992 / 1997 \AA$ triplet is in emission in contrast to weak absorption lines seen in the observation (Fig. 5). From the Ne VIII lines, no clear preference for the lower or higher $T_{\text {eff }}$ can be derived from the $1162-1166 \AA$ features. The $2821 / 2861 \AA$ doublet poses difficulties. The lines are not deep enough in the models. Figure 6 (bottom) shows that at $\mathrm{Ne}=1 \%$, neither a $180000 \mathrm{~K}$ model nor a $200000 \mathrm{~K}$ model fits. A $170000 \mathrm{~K}$ model (not shown) has marginally stronger profiles. This low temperature is, however, excluded because numerous strong Ne VII lines appear in that model that are not observed. Also, it is at odds with the lower $T_{\text {eff }}$ limit implied by the absence of O V $1371 \AA$. Figure 6 (top) shows that an increase in the $\mathrm{Ne}$ abundance to

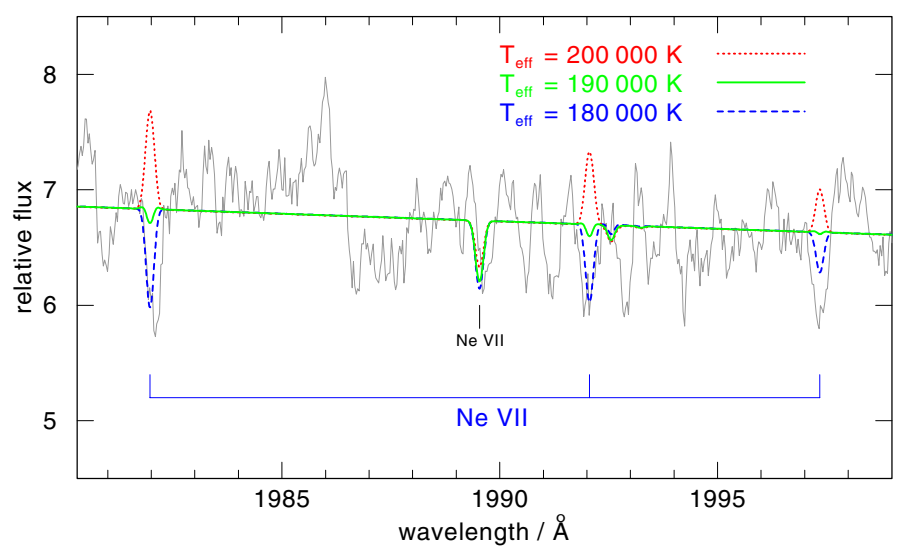

Fig. 5. Spectrum detail (thin gray line) comprising a $T_{\text {eff }}$ sensitive Ne VII triplet. Overplotted are three models (thick lines) with different $T_{\text {eff }}$ (solid, dashed, and dotted, as indicated in the legend). Other model parameters: $\log g=6.7, \mathrm{Ne}=1.2 \times 10^{-2}$. Observation and models were convolved with a $0.13 \AA$ boxcar and $0.15 \AA$ Gaussians, respectively.

$5 \%$ would be able to fit the observation, but then again, strong $\mathrm{Ne}$ VII lines appear that are not observed. Another problem arises with the $1932 \AA$ line, whose emission height is not at all achieved by any model. In conclusion, all the observed neon lines cannot be fit with a unique abundance value. We adopt $\mathrm{Ne}=1 \%$ as a compromise.

\subsection{Magnesium}

We searched for Mg lines without success. According to our models, Mg VII has the strongest lines, but wavelength positions are not known better than about $1-2 \AA$. At a solar abundance level, UV lines of detectable strength are predicted, most prominently the components of $\mathrm{a}^{3} \mathrm{P}^{\mathrm{O}}-{ }^{3} \mathrm{P}$ triplet at $1291-1350 \AA$. Some of the unidentified lines could therefore stem from this ion.

\subsection{Silicon}

The Si VII lines were identified in the FUSE spectrum of KPD $0005+5106$, and $\mathrm{Si}=1.3 \times 10^{-3}$ (twice solar) was derived 


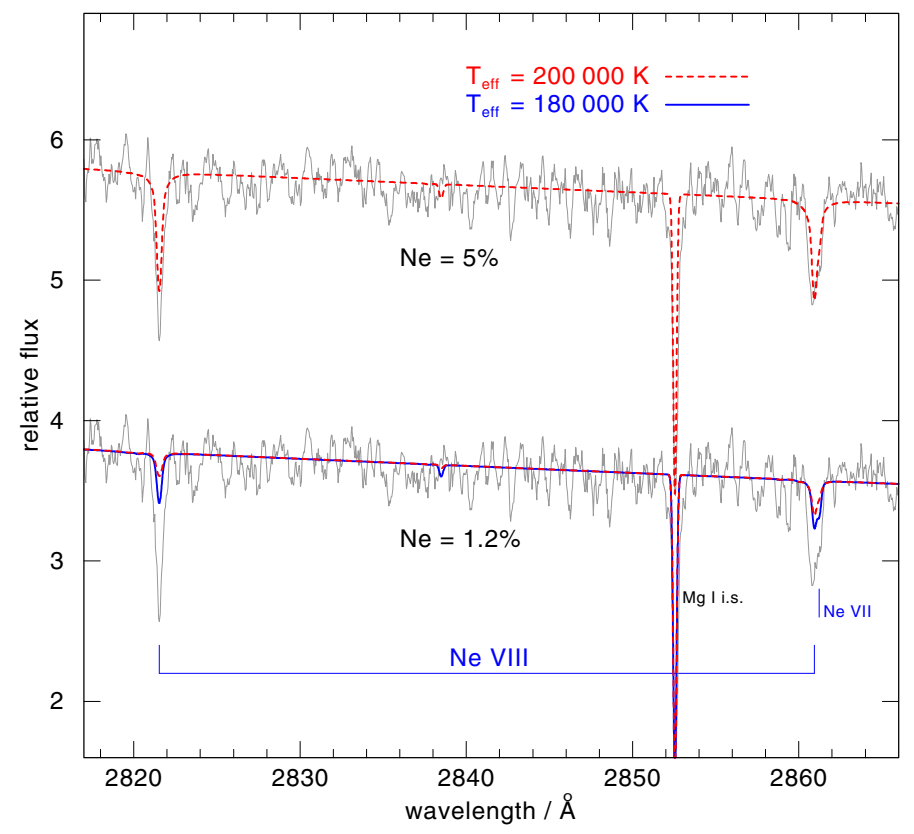

Fig. 6. Detail of the NUV spectrum (thin gray lines) showing the Ne VIII $3 s-3 p$ doublet. Bottom: overplotted are two models (thick lines) with different temperatures (dashed and solid lines as indicated in the legend) and $\mathrm{Ne}=1.2 \%$. Top: same spectrum and a model with $\mathrm{Ne}=5 \%$ (thick dashed line). Models have $\log g=6.7$. Observation and models were convolved with a $0.18 \AA$ A boxcar and $0.20 \AA$ Aaussians, respectively.

(Wassermann et al. 2010). Lines from this ion are also detectable in the STIS spectrum, e.g., at 1167.8 and $1169.3 \AA$, the two strongest components of a triplet (Fig. 1). A singlet at $1236.0 \AA$ (Kentucky database wavelength with uncertainty of $0.65 \AA$ ) is visible in the HST/GHRS data and was already assigned to Si VII (but not fit) by Wassermann et al. (2010). It is odd that the line profile observed in the STIS data is much broader and weaker than in the GHRS data (top inset in Fig. 3) and the computed profile. Si lines from adjacent ionization stages were not found in the FUSE spectrum, which was only compatible with a $200000 \mathrm{~K}$ model. The same holds for the STIS data. The $180000 \mathrm{~K}$ model shows prominent Si VI lines, while the $220000 \mathrm{~K}$ model exhibits Si VIII lines, while both ionization stages are not detectable in the observation (Fig. 3). With the quoted $\mathrm{Si}$ abundance, our model gives lines that are too strong: see the Si VII triplet displayed in Fig. 1. A good fit is obtained with $\mathrm{Si}=1.7 \times 10^{-4}$.

\subsection{Sulfur}

Lines of S VI and S VII were found in the FUSE spectrum (Wassermann et al. 2010). No sulfur lines can be identified in the STIS spectra, in accordance with our model predictions.

\subsection{Calcium}

Two CaX emission lines of the $4 \mathrm{p}-4 \mathrm{~d}$ doublet at 1136.5 and 1159.2 $\AA$ were discovered in the FUSE spectrum (Werner et al. 2008). The latter is covered by our STIS spectrum, but it cannot be identified because of the low $\mathrm{S} / \mathrm{N}$ in this region. In that paper, two absorption lines at 1461.2 and $1503.6 \AA$ in the IUE spectrum of the hot PG 1159 star NGC 246 were tentatively identified as the $4 s-4 p$ doublet. No features are seen in the STIS spectrum of KPD $0005+5106$ at these wavelengths.
Table 4. Abundances in KPD $0005+5106\left(\beta_{i}\right)$ and in the $\operatorname{Sun}\left(\beta_{\odot}\right)$.

\begin{tabular}{crrrc}
\hline \hline Element & $\log \beta_{i}$ & $\log \beta_{\odot}$ & $\log \left(\beta_{i} / \beta_{\odot}\right)$ & Reference \\
\hline $\mathrm{H}$ & $<-1.6$ & -0.1 & $<-1.5$ & $(1)$ \\
$\mathrm{He}$ & -0.01 & -0.6 & 0.6 & $(1)$ \\
$\mathrm{C}$ & -2.0 & -2.6 & 0.6 & $(1)$ \\
$\mathrm{N}$ & -2.6 & -3.2 & 0.6 & $(1)$ \\
$\mathrm{O}$ & -2.4 & -2.2 & -0.2 & $(1)$ \\
$\mathrm{Ne}$ & -2.0 & -2.9 & 0.9 & this work \\
$\mathrm{Mg}$ & $<-3.2$ & -3.2 & $<0.0$ & this work \\
$\mathrm{Si}$ & -3.8 & -3.2 & -0.6 & this work \\
$\mathrm{S}$ & -3.1 & -3.5 & 0.4 & $(1)$ \\
$\mathrm{Ca}$ & -4.2 & -4.2 & 0.0 & $(2)$ and this work \\
$\mathrm{Fe}$ & -2.9 & -2.9 & 0.0 & $(1)$ \\
\hline
\end{tabular}

Notes. Abundances by mass fraction. Solar abundances from Asplund et al. (2009).

References. (1) Wassermann et al. (2010); (2) Werner et al. (2008). ${ }^{(a)}$ In this work, only an upper limit was determined.

In the $T_{\text {eff }}$ range of $170000-220000 \mathrm{~K}$ covered by our models, the $\mathrm{CaX} 4 \mathrm{~s}-4 \mathrm{p}$ lines become weaker with increasing temperature. At solar $\mathrm{Ca}$ abundance $\left(\mathrm{Ca}=6.4 \times 10^{-5}\right)$, the lines become undetectable at $T_{\text {eff }}=200000 \mathrm{~K}$, confirming the solar abundance value derived from the $4 \mathrm{p}-4 \mathrm{~d}$ doublet by Werner et al. (2008), while models with the about four times higher value derived by Wassermann et al. (2010) predict lines strong enough to be detectable. The model with $T_{\text {eff }}=180000 \mathrm{~K}$ and solar $\mathrm{Ca}$ abundance predicts detectable lines so that at this temperature only a one-third solar $\mathrm{Ca}$ abundance would explain the observation.

\subsection{Iron}

A solar abundance $\left(\mathrm{Fe}=1.3 \times 10^{-3}\right)$ was derived from $\mathrm{Fe} \mathrm{X}$ lines in the FUSE spectrum (Wassermann et al. (2010). The star is too hot to exhibit Fe VII or Fe VIII lines (Werner et al. 2011). No iron lines are available in the wavelength range of our STIS spectra.

\subsection{Unidentified lines}

As mentioned, some of the unidentified lines probably stem from $\mathrm{Ne}$ VII and Mg VII. We checked the line lists cited above for other possible candidates but found no plausible identifications. For our search we looked for lines from light metals heavier than CNO in ionization stages VI-XI and wavelengths known with an accuracy of at least about $0.5 \AA$ and - if available - high $g f$ values.

\section{Summary and discussion}

The analysis of the metal lines in the new HST spectra of KPD $0005+5106$ confirms the result of Wassermann et al. (2010), who found $T_{\text {eff }}=200000 \pm 20000 \mathrm{~K}$. Our investigation of the ionization balances of neon and silicon yields a compatible result with a slightly smaller error, namely $T_{\text {eff }}=$ $195000 \pm 15000 \mathrm{~K}$. Therefore, the metal abundances determined by Wassermann et al. (2010) are confirmed with the exception of neon and silicon, which we improved, and an upper limit for $\mathrm{Mg}$ was determined. The element abundances are summarized in Table 4 and displayed in Fig. 7, together with results for RCB and EHe stars (from Jeffery et al. 2011, and references 


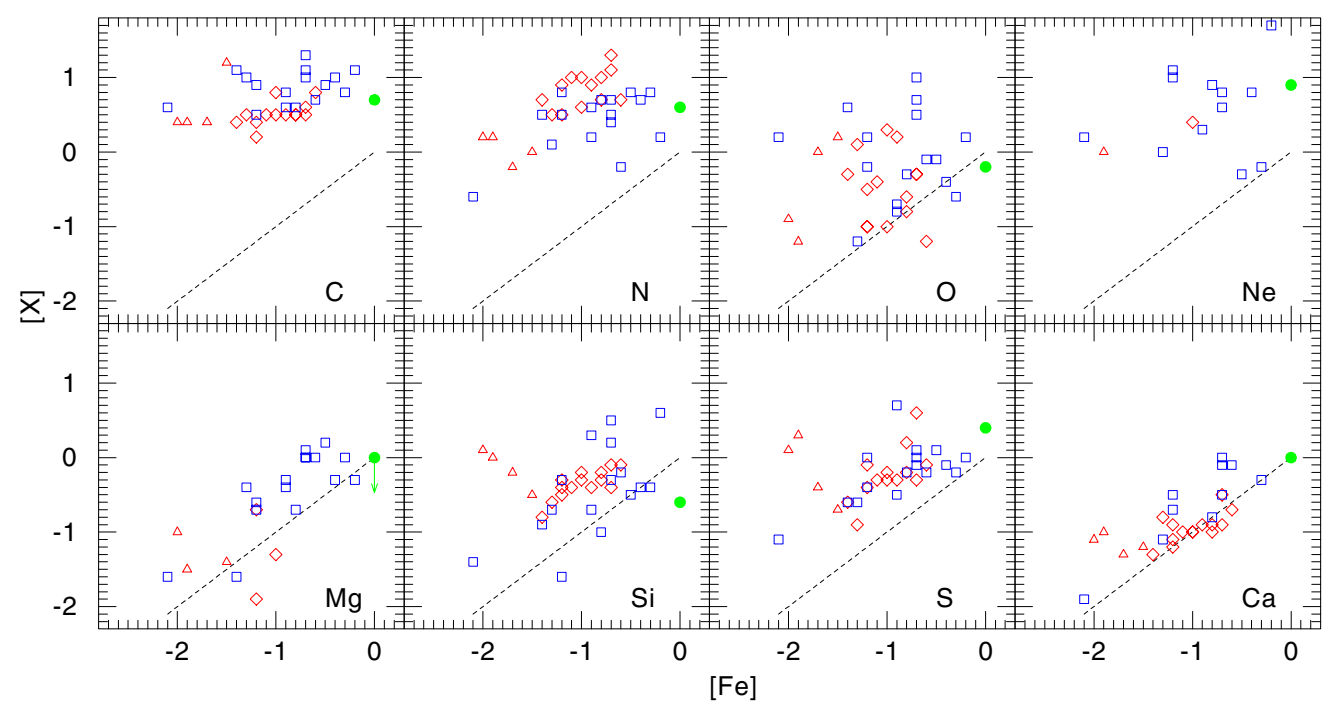

Fig. 7. Observed abundances (log number relative to solar; one panel per species) versus iron abundance (same units) for EHe stars (blue squares), majority RCB stars (red diamonds), minority RCB stars (red triangles; from Jeffery et al. 2011, and references therein) and KPD $0005+5106$ (green filled circle). The upper limit for $\mathrm{Mg}$ is indicated by an arrow. The dashed lines indicate the solar composition scaled to iron.

therein $)^{8}$. Typical errors are about 0.5 dex for KPD $0005+5106$ and 0.3 dex for the RCB and EHe stars. It is obvious that the metal abundances in KPD $0005+5106$ are very similar to RCB and EHe stars.

Currently, ten objects were assigned to the group of $\mathrm{O}(\mathrm{He})$ stars (Reindl et al. 2014; Werner et al. 2014; De Marco et al. 2015). They cover a parameter range of $T_{\text {eff }}=$ $80000-195000 \mathrm{~K}$ and $\log g=5.0-6.7$. (KPD $0005+5106$ is the hottest member and, together with the central star of the planetary nebula $\mathrm{Pa}$ 5, has the highest gravity.) Reindl et al. (2014) noticed that they fall into three subgroups: C-rich, N-rich, and $\mathrm{C}+\mathrm{N}$-rich. It was argued that this can be explained by different scenarios within a He-WD + He-WD merger (so-called fastmerger, slow-merger, and composite merger) for which abundance predictions were made by evolution model calculations by Zhang \& Jeffery $(2012 \mathrm{a}, \mathrm{b})$ to show that RCB stars and helium-rich subdwarf $\mathrm{O}$ stars could be formed by a binary $\mathrm{He}-\mathrm{WD}$ merger. Only two $\mathrm{O}(\mathrm{He})$ stars fall into the latter group: SDSS J172854.34+361958.62 (henceforth SDSS J1728; Werner et al. 2014) and KPD 0005+5106, leading Reindl et al. (2014) to conclude that they are the result of a double He-WD composite merger that descended from an RCB star. With $T_{\text {eff }}=$ $100000 \mathrm{~K}$ and $\log g=5.0$, SDSS J1728 is located halfway between the RCB stars and KPD 0005+5106 in the Hertzsprung Russell Diagram.

However, based on arguments from binary population synthesis, only $1 \%$ of the RCB stars may form from double $\mathrm{He}-\mathrm{WDs}$, and the majority forms from a He-WD + COWD merger (see Zhang et al. 2014, and references therein). In the cited work, it is demonstrated that post-merger evolution calculations predict surface abundances that can partially explain the observations in RCB stars, in particular for the elements studied in KPD 0005+5106 in the present paper. A comparison of the results for KPD 0005+5106 confirms earlier suggestions (Rauch et al. 2008; Wassermann et al. 2010) that KPD $0005+5106$ is indeed an evolved RCB star.

\footnotetext{
8 Numerical differences between abundances of RCB and EHe stars in our figure and the respective Fig. 1 in Jeffery et al. (2011) stem from our using different values for the solar element abundances, i.e., Asplund et al. (2009) instead of Anders \& Grevesse (1989).
}

The calculations of Zhang et al. (2014) show that the observed $\mathrm{C}$ abundance in RCB stars can only be produced by CO WDs in a very narrow mass range $\left(0.55 \pm 0.02 M_{\odot}\right)$ merging with a He-WD with a mass in the range $0.3-0.45 M_{\odot}$. The total mass should therefore be in the range $0.85-1.1 M_{\odot}$, which is similar to observed masses of RCB stars, deduced from the luminosity and evolution calculations $\left(0.8-1.0 M_{\odot}\right)$. The masses of the two $\mathrm{C}+\mathrm{N}$-rich $\mathrm{O}(\mathrm{He})$ stars are $0.64_{-0.04}^{+0.08} M_{\odot}$ and $0.73_{-0.12}^{+0.14} M_{\odot}$ for KPD $0005+5106$ and SDSS J1728, respectively. While the mass of SDSS J1728 is relatively high, that of KPD 0005+5106 appears too low, suggesting its origin is either one of the rare $\mathrm{He}+\mathrm{He}$ WD mergers or a $\mathrm{He}+\mathrm{CO}$ WD merger where the $\mathrm{He}$ WD had a significantly lower mass than the lower $0.3 M_{\odot}$ limit postulated by Zhang et al. (2014). But we do note that the masses of the two stars were derived with VLTP post-AGB tracks, while post-merger tracks yield masses that are systematically higher by about $0.1-0.2 M_{\odot}$ (Reindl et al. 2014).

In the context of the $\mathrm{O}(\mathrm{He})$ stars it is also worth emphasizing the finding by Zhang et al. (2014) that CO+He WD mergers may also result in stars that are not carbon rich. Thus one could conceive that many more of the $\mathrm{O}(\mathrm{He})$ stars are the result of such mergers and not of $\mathrm{He}+\mathrm{He}$ WD mergers, although their masses appear too low.

To conclude, we confirm the general picture of hot heliumdominated pre-white dwarfs as the result of binary WD mergers; however, the detailed nature of the original binary systems remains unclear.

Acknowledgements. T. Rauch had been supported by the German Aerospace Center (DLR) under grant 05 OR 1301. This research made use of the SIMBAD database, operated at the CDS, Strasbourg, France, and of NASA's Astrophysics Data System Bibliographic Services. Some of the data presented in this paper were obtained from the Mikulski Archive for Space Telescopes (MAST). This work used the profile fitting procedure Owens developed by M. Lemoine and the FUSE French Team.

\section{References}

Anders, E., \& Grevesse, N. 1989, Geochim. Cosmochim. Acta, 53, 197 Asplund, M., Grevesse, N., Sauval, A. J., \& Scott, P. 2009, ARA\&A, 47, 481 Bashkin, S., \& Stoner, J. O. 1975, Atomic energy levels and Grotrian Diagrams Vol.1: Hydrogen I - Phosphorus XV; Vol. 2: Sulfur I - Titanium XXII Clayton, G. C. 1996, PASP, 108, 225 
Clayton, G. C., Geballe, T. R., Herwig, F., Fryer, C., \& Asplund, M. 2007, ApJ, 662,1220

De Marco, O., Long, J., Jacoby, G. H., et al. 2015, MNRAS, 448, 3587

Dere, K. P., Landi, E., Mason, H. E., Monsignori Fossi, B. C., \& Young, P. R. 1997, A\&AS, 125, 149

Downes, R. A., Sion, E. M., Liebert, J., \& Holberg, J. B. 1987, ApJ, 321, 943

Hébrard, G., \& Moos, H. W. 2003, ApJ, 599, 297

Hébrard, G., Friedman, S. D., Kruk, J. W., et al. 2002, Planet. Space Sci., 50, 1169

Herwig, F., Blöcker, T., Langer, N., \& Driebe, T. 1999, A\&A, 349, L5

Iben, Jr., I. 1995, Phys. Rep., 250, 2

Iben, Jr., I., Kaler, J. B., Truran, J. W., \& Renzini, A. 1983, ApJ, 264, 605

Jeffery, C. S., Karakas, A. I., \& Saio, H. 2011, MNRAS, 414, 3599

Kruk, J. W., \& Werner, K. 1998, ApJ, 502, 858

Landi, E., Young, P. R., Dere, K. P., Del Zanna, G., \& Mason, H. E. 2013, ApJ, 763,86

Mendez, R. H. 1991, in Evolution of Stars: the Photospheric Abundance Connection, eds. G. Michaud, \& A. V. Tutukov, IAU Symp., 145, 375

Pandey, G., Lambert, D. L., Jeffery, C. S., \& Rao, N. K. 2006, ApJ, 638, 454

Rauch, T., Reiff, E., Werner, K., \& Kruk, J. W. 2008, in Hydrogen-Deficient Stars, eds. A. Werner, \& T. Rauch, ASP Conf. Ser., 391, 135

Reader, J., Kramida, A., Ralchenko, Y., Wiese, W., \& Fuhr, J. 2015, J. Phys. Conf. Ser., 576, 012007
Reindl, N., Rauch, T., Werner, K., Kruk, J. W., \& Todt, H. 2014, A\&A, 566, A 116

Saio, H., \& Jeffery, C. S. 2002, MNRAS, 333, 121

Seaton, M. J., Yan, Y., Mihalas, D., \& Pradhan, A. K. 1994, MNRAS, 266, 805 Sion, E. M., \& Downes, R. A. 1992, ApJ, 396, L79

Wassermann, D., Werner, K., Rauch, T., \& Kruk, J. W. 2010, A\&A, 524, A9

Webbink, R. F. 1984, ApJ, 277, 355

Werner, K., \& Dreizler, S. 1999, J. Comput. Appl. Math., 109, 65

Werner, K., \& Herwig, F. 2006, PASP, 118, 183

Werner, K., Heber, U., \& Fleming, T. 1994, A\&A, 284, 907

Werner, K., Dreizler, S., Heber, U., et al. 1996, A\&A, 307, 860

Werner, K., Deetjen, J. L., Dreizler, S., et al. 2003, in Stellar Atmosphere Modeling, eds. I. Hubeny, D. Mihalas, \& K. Werner, ASP Conf. Ser., 288, 31

Werner, K., Rauch, T., \& Kruk, J. W. 2007, A\&A, 474, 591

Werner, K., Rauch, T., \& Kruk, J. W. 2008, A\&A, 492, L43

Werner, K., Rauch, T., Kruk, J. W., \& Kurucz, R. L. 2011, A\&A, 531, A146

Werner, K., Dreizler, S., \& Rauch, T. 2012, Astrophysics Source Code Library [record ascl:1212.015]

Werner, K., Rauch, T., \& Kepler, S. O. 2014, A\&A, 564, A53

Zhang, X., \& Jeffery, C. S. 2012a, MNRAS, 426, L81

Zhang, X., \& Jeffery, C. S. 2012b, MNRAS, 419, 452

Zhang, X., Jeffery, C. S., Chen, X., \& Han, Z. 2014, MNRAS, 445, 660 